\title{
Device Complication
}

National Cancer Institute

\section{Source}

National Cancer Institute. Device Complication. NCI Thesaurus. Code C78254.

Any problem arising from the use of a medical device. 